\title{
Effectiveness of dexmedetomidine for delirium and its impact on hemodynamics in critically ill patients
}

\author{
Shamim Kausar, Iqra Yasin $\triangle$, Irfan Ahsan $\triangle$, Zunairah Rais $\square$ \\ Author affiliations: \\ MICU, Liaquat National Hospital, National Stadium Road, Karachi City 74800, Karachi, Pakistan. \\ Correspondence: Dr. Shamim Kausar, A-351 Rafa-e-Aam Society, Malir Halt, Karachi, Pakistan; E-mail: \\ drshamimahsan@gmail.com; Phone: 03343093627
}

\section{Abstract}

Aim/Background: Delirium is common in critically ill patients. For its treatment along with other sedatives, dexmedetomidine has also been prescribed by the clinicians. However, the outcomes with its use are less well-defined by any study in local population. We aimed to study its effectiveness for delirium and the impact on hemodynamics in critically sick patients of our population.

Methodology: It was a cross-sectional, prospective observational study conducted on 212 participants after ethical review committee approval at our hospital. Heart rate, systolic and diastolic blood pressures and Richmond Agitation Sedation Scale (RASS) scores were recorded before starting dexmedetomidine and within one hour of starting the drug. More than $10 \%$ decrease in heart rate (HR) or systolic or diastolic blood pressure was considered as an impact on hemodynamic parameters. An achievement of RASS of -2 was considered as effectiveness of drug.

Results: There was a mean drop of HR by $13.78 \pm 18.58$ beats/min (14.49\%) after starting dexmedetomidine. Mean systolic blood pressure drop was $10.99 \pm 20.67 \mathrm{mmHg}$ (9.16\%), and mean drop in diastolic blood pressure was $4.958 \pm 12.53 \mathrm{mmHg}$ after dexmedetomidine use. The mean value of RASS score achieved on dexmedetomidine was -2 .

Conclusion: Our study concluded the effectiveness of dexmedetomidine as light sedation for delirium. It significantly impacted on hemodynamic parameters. Its impact on heart rate was more pronounced as compared to systolic and diastolic blood pressures.

Key words: Dexmedetomidine; Critical care; Delirium; Hemodynamics

Citation: Kausar S, Yasin I, Ahsan I, Rais Z. Effectiveness of dexmedetomidine for delirium and its impact on hemodynamics in critically ill patients. Anaesth. pain intensive care 2020;24(6):645-649; DOI: 10.35975/apic.v24i6.1402

Received: 14 June 2020, Reviewed: 24 August, 19 October 2020, Accepted: 26 October 2020

\section{Introduction}

Delirium is a state of various behavioral changes like fluctuating mental status, agitation and altered sensorium, and it is quite common amongst critically ill patients. Timothy D Girard et al. ${ }^{1}$ conducted a multicenter prospective cohort study and found that Delirium affected $71 \%$ of participant and sedative induced delirium showed the worst outcomes in terms of declining cognitive function amongst other phenotype of hypoxic delirium, septic delirium, unclassified and metabolic delirium.
There are other risk factors including advanced age and higher SOFA score, ${ }^{2}$ regular smoking and exposure to high doses of midazolam. However, sequential sedation with dexmedetomidine decreased the risk of delirium. ${ }^{3}$ Mayur B. Patel et al. assessed delirium in neurocritically ill patients and found a frequency of delirium to be $12-43 \%$. Delirium was associated with increased hospital length of stay and ICU days. ${ }^{4}$ Various tools are used to diagnose delirium, like The Confusion Assessment Method for the ICU (CAM-ICU) and arousal scale. Study reviewed the epidemiology, current treatment being offered to critically ill patients and further research 
opportunity and performance of different ICU delirium prediction models. ${ }^{5,6}$ However in many intensive care units (ICU) international guidelines for sedation and delirium are not practiced. ${ }^{7}$ A metaanalysis showed that multi-component implementation programs for assessment of delirium its prevention and treatment can improve clinical outcomes. $^{8}$

Use of dexmedetomidine is helpful in prevention and treatment of delirium. It's an alpha2 receptor agonist and has no anticholinergic activity so natural sleep cycle is not disturbed. It causes inhibition of norepinephrine by acting as $\alpha_{2}$-adrenoceptors agonist on locus cereulus of pons. ${ }^{2}$ Cerebral cortical activity is significantly altered in various brain areas during sedation with dexmedetomidine. ${ }^{9}$ The role of dexmedetomidine is established for sedation level not deeper than arousal on verbal stimulation response and it is not suitable for deeper sedation. Post-operative scheduled acetaminophen combined with dexmedetomidine reduced in hospital delirium as compared to placebo group. ${ }^{10}$, Variable results of dexmedetomidine in adult ICU for sedation have been shown. ${ }^{11,12,13}$ Patients are calm, cooperative and easily arousable on dexmedetomidine. Patients do not develop respiratory depression, ${ }^{2}$ and unwanted sedative induced invasive ventilations are avoided. However its use is associated with bradycardia, ${ }^{14}$ and hypotension. Its peripheral vasoconstriction effect is concentration dependent. ${ }^{15}$ It may reduce the duration of mechanical ventilation and need of tracheostomy while there was an increase in frequency and duration of ICU delirium. ${ }^{16,17}$

We aimed to determine the effectiveness of dexmedetomidine for delirium and its impact on hemodynamics in critically ill patients of our population.

\section{Methodology}

The study was a cross sectional, prospective study, conducted from 20th March 2020 to 5th May 2020 after approval of ethical committee, in Medical ICU and Medical High Dependency Unit (HDU) of Liaquat National Hospital and Medical College. The sample size of 232 was calculated using the World Health Organization (WHO) software, taking the prevalence of decrease in systolic blood pressure (SBP) after drug $=16.5 \%,{ }^{18}$ margin of error $=5 \%$ and confidence level
$=95 \%$. Samples were collected in a non-probability consecutive sampling manner. All patients admitted in medical HDU and ICU for more than $12 \mathrm{hrs}$ fulfilling inclusion criteria, including age $>14 \mathrm{yrs}$, delirium CAM-ICU criteria, sedation used for more than $4 \mathrm{hrs,}$ intubated and non-intubated were enrolled after taking informed consent. Patient with a history of substance abuse, acute cerebrovascular accident, heart rate (HR) $<60$ beats/min, were excluded from the study.

Patient history including their age and gender was noted. Delirium was confirmed by CAM-ICU scale. Agitation and sedation were graded using Richmond agitation sedation scale (RASS) before starting sedation and at $1 \mathrm{hr}$ after starting the sedation. The loading dose of dexmedetomidine was not given. Systolic blood pressures (SBP) and diastolic blood pressures (DBP) were recorded by invasive blood pressure recorder through arterial line and HR was recorded through continuous $\mathrm{HR}$ monitor before starting sedation and minimum values were noted on sedation. Confounding variables and biases were controlled by strictly following the inclusion and exclusion criteria. Data were recorded in a proforma.

Statistical analysis: Data were analyzed by using SPSS version 20. Mean and standard deviation values were computed for quantitative variable i.e. age, RASS score, systolic and DBP, HR before and after sedation, maximum maintenance dose given and RASS before sedation and on sedation. Frequency and percentage were calculated for qualitative variables i.e. gender, initial diagnosis, BP ( $\max / \mathrm{min}), \mathrm{HR}$ ( $\max / \mathrm{min})$ and intervention required to control hemodynamic instability (sedation stopped/atropine or dopamine given/vasopressor started). Effectiveness of dexmedetomidine was defined by targeting RASS of 2 on dexmedetomidine. Impact on hemodynamics was concluded by considering either $10 \%$ fall in $\mathrm{HR}$, or in systolic or DBP after drug.

\section{Results}

Mean age of the patient was $49.46 \pm 19.49 \mathrm{yr}$. There were $95(44.8 \%)$ males and $117(55.2 \%)$ female patients. Out of 212 patients, 110 (51.9\%) were intubated. The mean maximum infused dose of dexmedetomidine was $0.7 \mu \mathrm{g} / \mathrm{kg} / \mathrm{hr}$, the range being $0.3-1.4 \mu \mathrm{g} / \mathrm{kg} / \mathrm{hr}$. 
After diagnosis of delirium by CAM-ICU scale the agitation was graded as per RASS before starting dexmedetomidine to be zero in $2(0.9 \%)$ patients, +1 by $39(18.4 \%),+3$ by $62(29.2 \%)$, while +4 was scored by $9(4.2 \%)$ patients.

The mean pre medication HR was $95.04 \pm 19.90$ beats/min. While the minimum value of mean HR post infusion of the drug was $81.25 \pm 18.31$, with a decrease of $13.78 \pm 18.579$ beats/min. This change equals a $14.49 \%$ decrease in HR. Mean value of pre medication
SBP was $120.85 \pm 20.11 \mathrm{mmHg}$ and post medication mean of minimum value of SBP was $109.86 \pm 18.29$. There was a mean fall of $10.99 \pm 20.67 \mathrm{mmHg}$ in blood pressure after the study drug which equals to a change of $9.16 \%$ decrease. The pre drug mean of DBP was $73.12 \pm 11.95 \mathrm{mmHg}$, while on drug infusion mean in DBP was $68.17 \pm 10.83$, with a difference of $4.96(6.7 \%) \mathrm{mmHg}$ fall. Mean RASS score before starting sedation was $+2.17 \pm 0.81$, reduced to $-2.00 \pm$ 0.97 after starting infusion of dexmedetomidine (Table 2).

Table 1: Maximum increase in HR and blood pressure on dexmedetomidine.

\begin{tabular}{l|c|c|c} 
Parameters & Pre dexmedetomidine & $\begin{array}{c}\text { Maximum values on } \\
\text { dexmedetomidine }\end{array}$ & $\begin{array}{c}\text { Minimum values on } \\
\text { dexmedetomidine }\end{array}$ \\
\hline HR $($ beats $/ \mathrm{min})$ & $95.04 \pm 19.90$ & $89.20 \pm 21.60$ & $81.25 \pm 18.31$ \\
\hline SBP $(\mathrm{mmHg})$ & $120.85 \pm 20.11$ & $116.02 \pm 16.09$ & $109.86 \pm 18.29$ \\
\hline DBP $(\mathrm{mmHg})$ & $73.12 \pm 11.95$ & $71.07 \pm 9.79$ & $68.17 \pm 10.83$ \\
\hline
\end{tabular}

$H R=$ heart rate, $S B P=$ Systolic blood pressure, $D B P=$ diastolic blood pressure

Table: 2 Frequency of different level of RASS achieved on dexmedetomidine.

\begin{tabular}{l|c}
\hline \multicolumn{1}{c}{$N(\%)$} & RASS \\
\hline $96(45.28)$ & -2 \\
\hline $67(31.60)$ & -3 \\
\hline $41(19.34)$ & -1 \\
\hline $5(2.36)$ & 0 \\
\hline
\end{tabular}

In $81(38.2 \%)$ of patients there was no need to stop sedation at any level due to its adverse hemodynamic issues. In $90(42.5 \%)$ of the patients sedation was stopped due to its impact as decrease in HR or BP. $12(5.7 \%)$ of the patients were continued on dexmedetomidine but also started on dopamine infusion or given atropine to increase HR. Vasopressors were started in $29(13.7 \%)$ of the patients to support falling blood pressure while dexmedetomidine infusion was not stopped.

\section{Discussion}

In our study there were more female patients as compared to males. The drug was mostly used in intubated patients due to different indications. The majority of the patient's score of agitation as per RASS was +2 before starting on dexmedetomidine while few of them reached a +4 scale of agitation. We used FDA recommended doses of dexmedetomidine and the mean RESS score on drug sedation was -2 , which was an effective and desired level of sedation.

On different maintenance doses of dexmedetomidine the RASS scores did not exceed beyond -3, as shown in Table 2. So it could not achieve a deeper sedation levels which makes it a less appropriate choice to be used in condition where deep sedation is required or a paralyzing agent is needed.

Dexmedetomidine impacted on HR by causing more than $10 \%$ mean drop in HR before starting medication and within $1 \mathrm{hr}$ of starting its infusion. Same conclusion was also derived by other authors in their study. ${ }^{3}$ Due to its significant impact on HR in majority of the patients the drug was stopped while some of them had to be infused dopamine infusion or given small doses of atropine to combat its effect on HR while it continued to have its sedative effect. There was a less than $10 \%$ fall in mean SBP before starting treatment and on treatment, so dexmedetomidine could not significantly impact on SBP as per defined criteria of impact on hemodynamics. However, based on individual need to support falling blood pressure, few of them were started on vasopressors to achieve target blood pressure. Dexmedetomidine impacted least on DBP in infusion. There are studies, ${ }^{1}$ that 
explain hypertension as an effect of dexmedetomidine but in our study mean of maximum recorded values did not prove any rise in HR and systolic or DBP on dexmedetomidine.

\section{Limitations}

This study is limited to the diagnosis of delirium in ICU, and does not discriminate the cause of delirium and effectiveness of dexmedetomidine in different types of delirium. Moreover, other factors impacting hemodynamics in critical care setting were not considered.

\section{Conclusion}

On the basis of our study, we conclude that dexmedetomidine is effective as sedative to reduce delirium in critical patients admitted in intensive care unit without significant impact on hemodynamic parameters.

\section{Future direction}

Dexmedetomidine can prove to be a leading sedative, whenever light sedative effects are required to control delirium in critical patients. However, more studies with larger sample size are needed to define its final outcome in critical care in terms of length of ICU stay, days on mechanical ventilation, sedation during weaning period, and mortalities.

\section{Authors' contribution}

SK: Conceptualization, original draft writing, analysis and methodology

IY: editing and data curation

IA: supervision, Project administration and writing review

ZR: data curation and methodology

\section{References}

1. Girard TD, Thompson JL, Pandharipande PP, Brummel $\mathrm{NE}$, Jackson JC, Patel MB, et al. Clinical phenotypes of delirium during critical illness and severity of subsequent long-term cognitive impairment: a prospective cohort study. Lancet Respir Med. 2018;6(3):213-222. [PubMed] DOI: 10.1016/S2213$\underline{2600(18) 30062-6}$
2. Scott-Warren VL, Sebastian J. Dexmedetomidine: its use in intensive care medicine and anaesthesia. BJA Edu. 2015;16(7):242-246. DOI: 10.1093/bjaed/mkv047

3. Yang J, Zhou Y, Kang Y, Xu B, Wang P, Lv Y, et al. Risk factors of delirium in sequential sedation patients in intensive care units. Bio Med Res Int. 2017;2017:1-9. [PubMed] DOI: 10.1155/2017/3539872

4. Patel MB, Bednarik J, Lee P, Shehabi Y, Salluh JI, Slooter AJ, et al. Delirium monitoring in neurocritically ill patients: a systematic review. Crit Care Med. 2018;46(11):1832-1841. [PubMed] DOI: 10.1097/CCM.0000000000003349

5. Pandharipande PP, Ely EW, Arora RC, Balas MC, Boustani MA, La Calle GH, et al. The intensive care delirium research agenda: a multinational, interprofessional perspective. Intensive Care Med. 2017;43(9):1329-1339. [PubMed] DOI: $\underline{10.1007 / s 00134-017-4860-7}$

6. Wassenaar A, Schoonhoven L, Devlin JW, van Haren FM, Slooter AJ, Jorens PG, et al. Delirium prediction in the intensive care unit: comparison of two delirium prediction models. Crit Care. 2018;22(1):114-123. [PubMed] DOI: 10.1186/s13054-018-2037-6

7. Kotfis K, Zegan-Barańska M, Żukowski M, Kusza K, Kaczmarczyk M, Ely EW. Multicenter assessment of sedation and delirium practices in the intensive care units in Poland-is this common practice in Eastern Europe? BMC Anesthesiol. 2017;17(1):120-130. [PubMed] DOI: 10.1186/s12871-017-0415-2

8. Trogrlić Z, van der Jagt M, Bakker J, Balas MC, Ely EW, van der Voort PH, Ista E. A systematic review of implementation strategies for assessment, prevention, and management of ICU delirium and their effect on clinical outcomes. Crit Care. 2015;19(1):157-174. [PubMed] DOI: 10.1186/s13054-015-0886-9

9. Kim WH, Cho D, Lee B, Song JJ, Shin TJ. Changes in brain activation during sedation induced by dexmedetomidine. J Int Med Res. 2017;45(3):11581167. [PubMed] DOI: $10.1177 / 0300060517705477$

10. Subramaniam B, Shankar $P$, Shaefi $S$, Mueller A, O'Gara B, Banner-Goodspeed V, et al. Effect of intravenous acetaminophen vs placebo combined with propofol or dexmedetomidine on postoperative delirium among older patients following cardiac surgery: the DEXACET randomized clinical trial. JAMA. 2019;321(7):686-696. [PubMed] DOI: 10.1001/jama.2019.0234

11. Deiner S, Luo X, Lin HM, Sessler DI, Saager L, Sieber $F E$, et al. Intraoperative infusion of dexmedetomidine for prevention of postoperative delirium and cognitive dysfunction in elderly patients undergoing major 
12. elective noncardiac surgery: a randomized clinical trial. JAMA Surg. 2017;152(8):1-8. [PubMed] DOI: 10.1001/jamasurg.2017.1505

13. Weatherall M, Aantaa R, Conti G, Garratt C, Pohjanjousi $P$, Lewis MA, et al. A multinational, drug utilization study to investigate the use of dexmedetomidine (Dexdor $\left.{ }^{\circledR}\right)$ in clinical practice in the EU. $\mathrm{Br} J$ Clin Pharmacol. 2017:83(9):2066-2076. [PubMed] DOl: 10.1111/bcp.13293

14. Kawazoe $Y$, Miyamoto $K$, Morimoto T, Yamamoto T, Fuke A, Hashimoto A, et al. Effect of dexmedetomidine on mortality and ventilator-free days in patients requiring mechanical ventilation with sepsis: a randomized clinical trial. JAMA. 2017;317(13):1321-1328. [PubMed] DOI: $\underline{10.1001 / j a m a .2017 .2088}$

15. Constantin JM, Momon A, Mantz J, Payen JF, De Jonghe $B$, Perbet $S$, et al. Efficacy and safety of sedation with dexmedetomidine in critical care patients: a meta-analysis of randomized controlled trials. Anaesth Critic Care Pain Med. 2016;35(1):7-15. [PubMed] DOI: 10.1016/j.accpm.2015.06.012
16. Talke P, Anderson BJ. Pharmacokinetics and pharmacodynamics of dexmedetomidine induced vasoconstriction in healthy volunteers. $\mathrm{Br} \mathrm{J}$ Clin Pharmacol. 2018;84(6):1364-1372. [PubMed] DOI: 10.1111/bcp.13571

17. Skrupky LP, Drewry AM, Wessman B, Field RR, Fagley $R E$, Varghese $L$, et al. Clinical effectiveness of a sedation protocol minimizing benzodiazepine infusions and favoring early dexmedetomidine: a before-after study. Crit Care. 2015;19(1):136-148. [PubMed] DOI: 10.1186/s13054-015-0874-0

18. Cruickshank M, Henderson L, MacLennan G, Fraser C, Campbell M, Blackwood B, et al. Alpha-2 agonists for sedation of mechanically ventilated adults in intensive care units: a systematic review. Health Technol Assess. 2016;20(25):1-118. [PubMed] DOI: 10.3310/hta20250

19. Nelson S, Muzyk AJ, Bucklin MH, Brudney S, Gagliardi JP. Defining the role of dexmedetomidine in the prevention of delirium in the intensive care unit. Bio Med Res Int. 2015;2015:1-7. [PubMed] DOI: 10.1155/2015/635737 